\title{
The Acoustic Characteristics Measurement and Evaluation on Underwater Complex Sound Sources
}

\author{
Dajing Shang ${ }^{1,2, a}$, Qi Li ${ }^{1,2}$, Rui Tang ${ }^{1,2}$, Hao Song ${ }^{2}$, Jundong Sun ${ }^{2}$ \\ ${ }^{1}$ Acoustic Science and Technology Laboratory, Harbin Engineering University, Harbin, China \\ ${ }^{2}$ College of Underwater Acoustic Engineering, Harbin Engineering University, Harbin, China \\ ae-mail:shangdajing@hrbeu.edu.cn
}

\begin{abstract}
Keywords: Underwater complex sound sources; Reverberation method; Radiated sound power measurement; Reverberation pool; Underwater propeller;
\end{abstract}

\begin{abstract}
Underwater complex sound sources have many types. The radiated power of these sound sources was obtained by measuring pressure in the ocean, but the measurement based on sound pressure in the ocean has some uncertainty. The sound pressure for one point or several points is not enough to characterize the sound sources. The acoustic measurement based on radiated power can reflect the acoustic characteristics of the sound sources exactly, especially the reverberation method can be used to measure the radiated power of underwater complex sound sources accurately in the reverberation pool and estimate the acoustic characteristics of the sound sources. First, the reverberation method measuring principle for underwater sound sources in the reverberation pool was described, the radiated power of a spherical standard sound source and an Underwater complex sound sources, such as underwater propeller was measured by reverberation method in the pool. Measurement and research results show that the difference between the radiated power of a standard spherical sound source measured by reverberation method and that measured in free field is not more than $1 \mathrm{~dB}$; The underwater complex sound source, such as underwater propeller can be measured by reverberation method accurately and the method can be also used to evaluate the radiated noise. The method can be applicable for investigating the acoustic characteristics of other underwater complex sound sources and also for the radiated sound power measurement and evaluation on underwater bodies under some condition.
\end{abstract}

\section{Introduction}

The characteristics of a sound source is an important acoustic research field, but it is difficult to measure, especially for the underwater sound source with complex structure. The sound field distribution for the underwater complex sound source is complicated, and the sound pressure for a point or points can not describe the sound source's characteristics fully. In order to describe the spatial characteristics of complex underwater sound source, it is necessary to know the spatial distribution of sound field caused by different sound source, but this is difficult for underwater complex sound sources, such as underwater ships. It is not easy to get the spatial characteristics of the sound source in the free field, let alone in the ocean (mostly shallow water area, the depth is only tens of meters, and exists the influence of sea surface and seabed, and the near field effects cannot be ignored also). Sound power can be used to reflect the acoustic characteristics of the sound source, and it is the function of frequency, it equals to the integration of sound intensity in each direction in the free field. The enveloping surface method is taken to get the sound power in a free field, it is necessary to get the sound intensity for each point on the enveloping surface, the radiated power of sound source can be gotten by integrating the sound intensity over the enveloping surface. The sound power can be measured in free-field pool for sound source with small size, but it is almost impossible to get the sound power for the large underwater structure in the same free-field pool.

Reverberation chamber is the standard measurement plant in air acoustics research. Sabine ${ }^{1}$ develop the reverberation method and was known as the father of the reverberation 
chamber, his diffusion field theory can be used to predict the diffusion field attenuation, reverberation time and steady-state sound pressure. The development of the reverberation chamber theory of air is developed, ${ }^{2-7}$ which is widely used to measure the sound power of complex irregular structure, noise source location, acoustic diagnosis and the measurement of random incidence sound absorption coefficient. In the reverberation chamber, G.C. Mailing ${ }^{8}$ calculated the radiated sound power of monopole source, Maidanik ${ }^{9}$ steadied stiffened plates by measuring the radiated sound power. Ludwig ${ }^{10}$ measured the radiated sound power of thin plate in the turbulence, G.M.Diehl ${ }^{11}$ measured a large machine by reverberation method. Under the same precision condition, the time to measure the radiated sound power of a complex sound source by reverberation method is $1 / 1000$ of the time required for free field method.

The underwater reverberation pool method is advised by the author ${ }^{12,13}$, which was used for measuring the radiated power of underwater model in water tunnel. After that, the reverberation method was applied to some domestic research and application for measuring small underwater target and high frequency sound source. In reverberant pool, Yu Mengsa ${ }^{14}$ measured the radiated sound power of a stiffened cylindrical shell model. Wang Chunxu ${ }^{15}$ measured the radiated sound power of underwater jet. The acoustic characteristics in a reverberant chamber or in a reverberant pool is different due to the larger difference between the physical characteristics of water and air. The boundary in reverberant chamber can be regarded rigid, but it might be regarded as soft or impedance boundary in reverberant pool; and the reflection coefficient of the wall in a reverberant pool is lower than that in a reverberation chamber. For this reason, it is difficult to establish an ideal underwater reverberation field in a pool, which is the main reason that the reverberation method is not suggested by more people. But the investigation of the author' team ${ }^{16,17}$ shows that reverberation method is still effective. This paper will further prove that the reverberation method can not only measure the radiated sound power of the simple sound source, but also measure the underwater complex sound sources and evaluate the characteristics of these sound sources.

\section{Principle of Reverberation method}

The characteristics of sound source can be expressed by the radiated power, which was measured by Reverberation method. The process of the radiated sound power measured by reverberation method was that we first measure spatial averaging sound pressure level, then the radiated power of the sound source can by gotten under the condition of reverberation pool calibration.

The sound source level measured by reverberation method can be expressed as ${ }^{18}$

$$
S L=\left\langle L_{P}\right\rangle-10 \log (R)
$$

$\mathrm{SL}(\mathrm{dB} r e 1 \mu \mathrm{Pa})$ is the source level, $\left\langle L_{P}\right\rangle$ ( $\mathrm{dB}$ re $\left.1 \mu \mathrm{Pa}\right)$ is the spatial averaging sound pressure level measured in reverberation control region, $10 \log (R)$ is the correction of reverberation field to the free field, which can also be expressed as:

$$
10 \log R=10 \log \left(\frac{8 \pi}{R_{0}}\right)
$$

In Eq. (2), $R_{0}=S\left(e^{\frac{55.2 \mathrm{~V}}{T_{60} S C_{0}}}-1\right) C_{0}$, where $\mathrm{S}$ is the area of the pool wall and bottom, $\mathrm{V}$ is the pool's volume, and $T_{60}$ is reverberation time. $10 \log (R)$ is the correction element, it is the difference between underwater acoustic source level and the spatial averaging sound pressure level measured in reverberation control area. The correction element is only related to the reverberation pool characteristics and can be gotten by measuring reverberant time.

The radiated power of a underwater complex sound sources can be gotten once the sound source level was measured by reverberation method. 


\section{The radiated power measurement for underwater complex sound sources by reverberation method}

\section{The measure system of reverberation method}

As a underwater complex sound source, the underwater propeller's radiated sound power measurement system was shown in Fig. 1.

The measuring system includes reverberation pool, underwater propeller structure and hydrophone array. The measurement was finished in the reverberation pool owned by the science and technology of underwater acoustic laboratory, Harbin Engineering University. The underwater propeller structure is fixed in the pool.

During measurement, the underwater propeller structure cannot be over the pool. Hydrophone array were arranged in the pool far from the propeller structure, but it can not be placed near the wall or the bottom of the pool. The spatial scan measurement was carried out by the hydrophone array's lateral moving along the platform (9 meters along the width direction) as was shown in Fig.1.

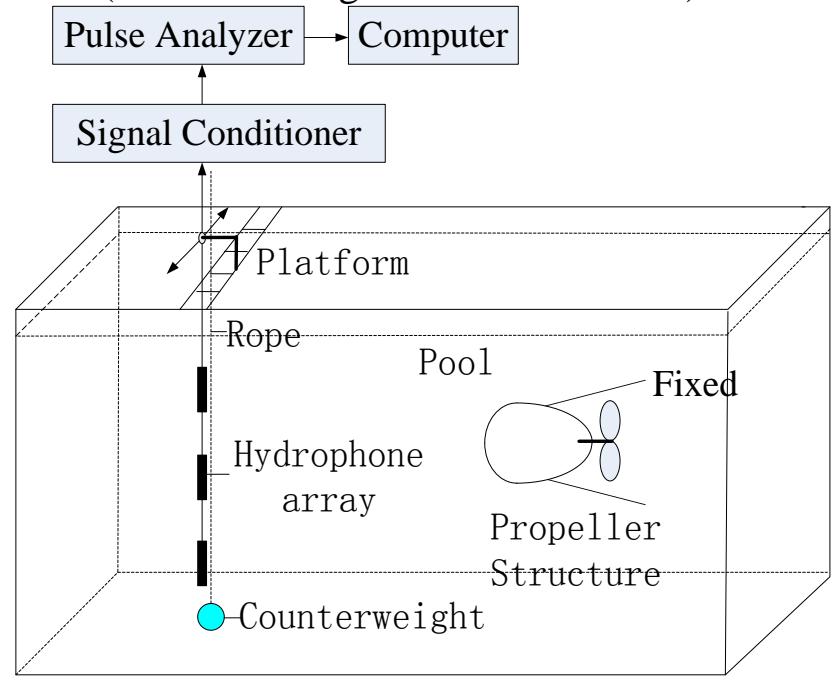

Fig.1 The measuring system for underwater complex sound sources

During the propeller running, in order to reduce the influence from flow, the acoustic permeable material can be used to isolate the hydrophone array. The spatial averaging sound pressure of propeller structure is measured by space average in the reverberation control region, the signal collected by hydrophone array is analyzed to filtered amplifier by intelligent conditioning (28000), and then sent to the PULSE dynamic signal analyzer as spectral analysis. In order to make the measuring points in the reverberation control region, the measuring points should be taken where far from the wall, the bottom of the pool and the source. While the hydrophone array's transversing scanning movement, we average hundreds times of sound pressure level by the analyzer, so that we can get a stable and repeatable spatial average sound pressure level signal.

The size of the pool is $15 \mathrm{~m} \times 9 \mathrm{~m} \times 6 \mathrm{~m}$, and the frequency range can be measured by reverberation method is $300 \mathrm{~Hz}-20 \mathrm{kHz}$; the major working frequency of the propeller structure is over $500 \mathrm{~Hz}$, so it is fit to measure the underwater sound source by reverberation method. 
The result Comparison for the standard sound source by reverberation method and free field method

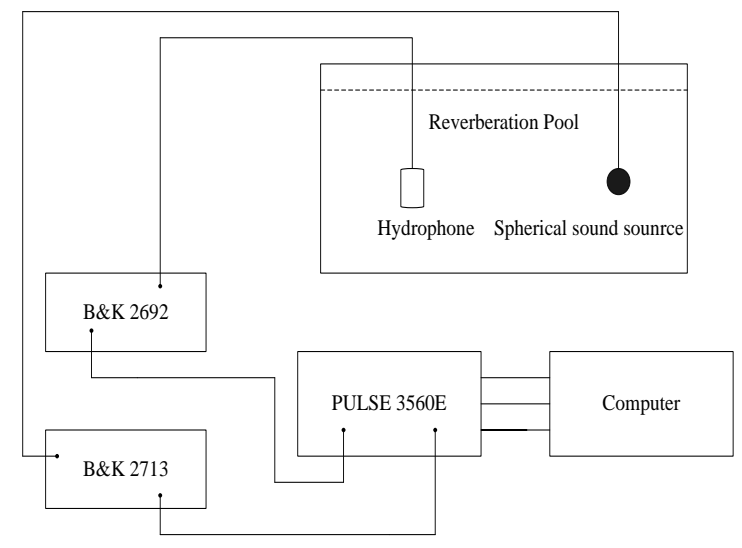

Fig.2 The reverberation method measuring system for a spherical sound source

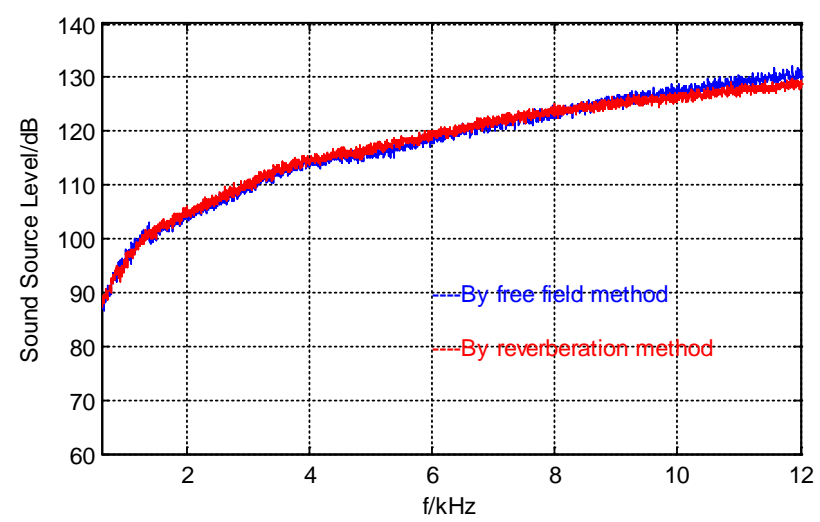

Fig.3 The comparison of the two methods

The sound source level of a standard spherical sound source was measured by reverberation method and free field method. The measuring system by reverberation method was shown in Fig.2. The white noise signal initiated by the PULSE(3560E) was amplified by the power amplifier(B\&K2713), and then was input to the standard sound source. The signals received by the hydrophone array were enlarged by the B\&K2692 and sent to the PULSE(3560E), then the radiated power of the standard source can be obtained. The results by different method were shown in Fig.3. It can be seen that the result difference between reverberation method and free field is not more than $1 \mathrm{~dB}$. It is proved that the radiated power of sound source can be measured accurately by reverberation method.

The measuring Results for underwater complex sound source by reverberation method

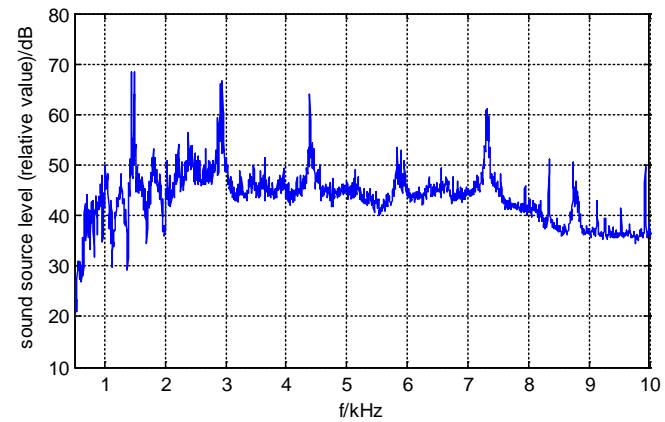

Fig.4 The measuring result for the underwater propeller in a cruising status.

The radiated noise for the underwater complex sound source was major shown as the line spectrum come from the motor of the propeller, its frequency was related to the speed of the motor 
and the wheel teeth number of the propeller. The frequency of the line spectrum for propeller in some status was $1448 \mathrm{~Hz}$, as shown in Fig.4; The meshing frequency of the propeller moter calculated was $1451 \mathrm{~Hz}$. The two results fits very well.

The radiated noise of the underwater propeller was measured in a reverberation pool by reverberation method, the underwater propeller model was shown in Fig.5

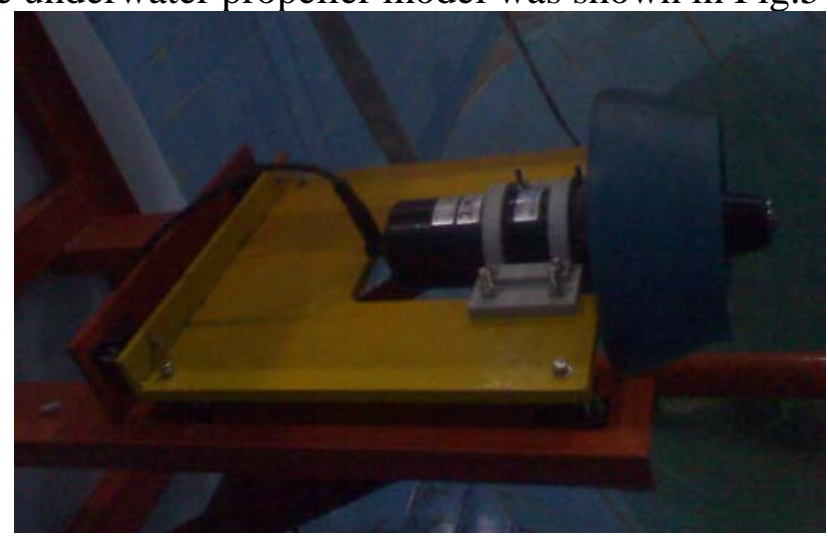

Fig.5 The underwater propeller

In the reverberation pool, the radiation noise of the propeller with and without vibration reduction equipment, was measured, the results were shown in Fig.8\& Fig.9. This showed that the reverberation method can be used to evaluate the noise of underwater complex sound sources so as to provide suggestion for the vibration control.

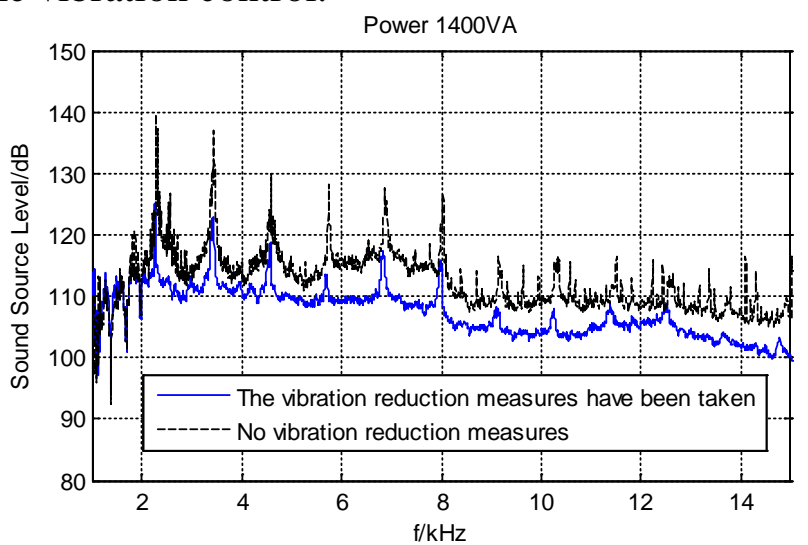

Fig.6 sound source level spectrum comparison

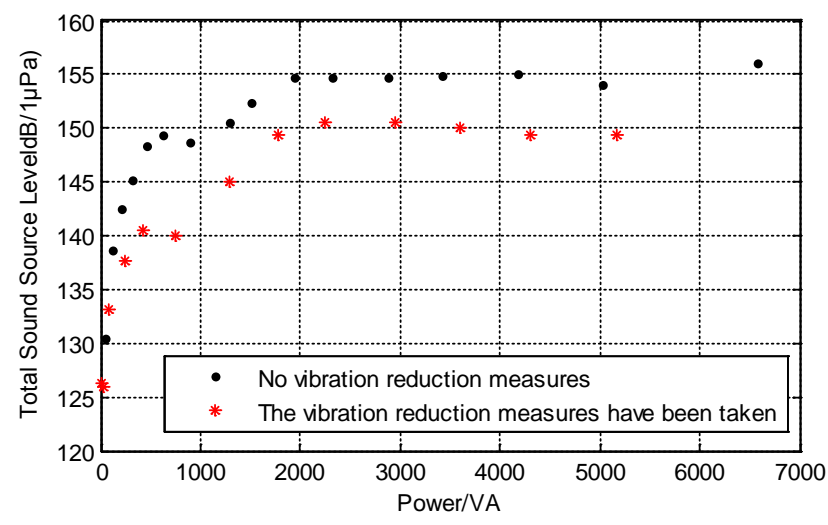

Fig.7 Total sound power level comparison 


\section{Summary}

The system for measuring the characteristics of underwater complex acoustic source was set up in the reverberation pool, and the radiated power of underwater complex sound sources like underwater propeller was measured by reverberation method by this system. The measuring results show that:

(1) The reverberant field characteristics of a reverberation pool is not as good as that of a reverberant chamber, but the results for the radiated power of underwater sound source measured are still effective, the results for the radiated power of underwater standard sound sources measured by reverberation method are almost same with that measured in the free field;

(2) The radiated power of an underwater complex sound source, such as underwater propeller can be measured by the reverberation method, the line spectrum frequency measured was equal to the meshing frequency of the propeller moter ;

(3) once the radiated power of the underwater complex sound source, such as underwater propeller, has been gotten by reverberant method, the noise level of this sound source can be evaluated and this can also give us some advises for reducing vibration and noise.

The research method in this paper can also be applied to other underwater sound sources with complex structures, such as underwater submarines.

\section{References}

[1] W. C. Sabine, Collected Papers on Acoustics (Dover, New York 1964), p. 245

[2] H. Kuttruff: Room Acoustics( Spon Press,London 2000)

[3] Maa Dah-You: Acta Acustica, Vol.28(2003),p.97

[4] Maa Dah-You: Acta Acustica, Vol.14(1989),p.383

[5] Maa Dah-You: Acta Acustica, Vol.19(1994), p.13

[6] Maa Dah-You: Acta Acustica, Vol.27(2002), p.385

[7] R. K. Cook, R. V. Waterhouse: J.Acoust. Soc.Am, Vol.27(1955),p.1072

[8] G. C. Mailing, JR: The Journal of the Acoustical Society of America, Vol.42(1967),p.859

[9] Maidanik, G: J.Acoust. Soc.Am, Vol.34(1962),p.809

[10] Ludwig, G. R: UTTA(Report 87, Univ. of Toronto)

[11]G. M. Diehl: J.Acoust. Soc.Am, Vol.61(1977),p.449

[12] Li Qi: Investigation on measurement method for water tunnel (Harbin shipbuilding engineering institute, Harbin1990)

[13]Li Qi, Yang Shie: Shipbuilding of china, Vol.3(1992),p.70

[14]Yu Mengsa, Lv shijin, Wu Yongxing: Applied Acoustics, Vol.20(2001),p.23

[15]Wang Chunxu, Zou Jian, Zhang Tao: Journal of Ship Mechnics, Vol.14(2010), p.172

[16] Shang Dajing, Li Qi, Shang Dejiang, Hou Benlong: Journal of Harbin Engineering University, Vol.31(7), (2010), p.938-944

[17] Shang Dajing, Li Qi, Shang Dejiang, Lin Han: Acta Acustica, Vol.37(2012), p.416

[18] Shang Dajing: Doctor Degree Paper(harbin engineering university, Harbin2012), p.45 